\title{
DIAGNÓSTICO DEL CLIMA ORGANIZACIONAL EN UNA EMPRESA
}

\author{
ATUNERA EN ECUADOR
}

\section{DIAGNOSIS OF THE ORGANIZATIONAL CLIMATE IN A TUNA \\ COMPANY IN ECUADOR}

\author{
Clara García ${ }^{1}$, Luis Valle ${ }^{2}$
}

\begin{abstract}
Palabras clave:
Clima laboral, Productividad, Trabajadores, Bienestar
\end{abstract}

\begin{abstract}
Resumen
Estudios previos han demostrado que, para mejorar los niveles de productividad en una empresa, es necesario crear un clima organizacional favorable. En ese sentido, se presenta el caso de estudio de una industria atunera en Ecuador que en los últimos años ha disminuido su productividad. Por tanto, el objetivo del presente estudio es determinar las dimensiones que intervienen en el clima organizacional de la empresa atunera ecuatoriana y que, a su vez, influyen en su productividad; mediante un cuestionario adaptado a la teoría de seis dimensiones del clima organizacional propuesta por Weisbord (1976), las cuales comprenden: propósitos u objetivos, relaciones entre los miembros, estructura y mecanismos de soporte de la gestión, mecanismos de apoyo, recompensas y liderazgo. Los resultados demuestran que, en efecto, existen puntos de mejora en cuanto a objetivos, liderazgo y sistema de recompensa, para los cuales se proponen algunas recomendaciones.
\end{abstract}

\section{Códigos JEL: M54}

Keywords:

Working

environment,

Productivity,

Employee,

Wellness

\footnotetext{
${ }^{1}$ Universidad Espíritu Santo (UUES), (Ecuador).

E-mail: clgarciar@uees.edu.ec

${ }^{2}$ Universidad Espíritu Santo (UEES), (Ecuador).

E-mail: luisvalle1103@gmail.com
} 
members, management support structure and mechanisms, support mechanisms, rewards and leadership. The results show that, in fact, there are improvement points in terms of objectives, leadership and reward system, for which some recommendations are proposed.

JEL Codes: M54

\section{INTRODUCCIÓN}

La aspiración de cualquier accionista o dueño de una empresa es obtener los mejores rendimientos en su productividad. Según Corredor (2016), para alcanzar un mejor rendimiento productivo, es necesario crear un buen clima laboral en el cual se satisfagan las aspiraciones tanto del empresario como de los trabajadores, lo que implica también crear un buen clima organizacional.

El clima organizacional consiste en priorizar y fortalecer las relaciones "sujeto-organización" y "sujeto-sujeto", dentro de cada uno de los departamentos que componen la entidad. Por la complejidad que dichas relaciones implican es que resulta un tema de mucho interés para múltiples profesionales y disciplinas. (Vega, Rodrigo, \& Partido, 2010).

Para Hall (2009), el clima organizacional es el conjunto de propiedades del ambiente laboral que los empleados pueden percibir directa o indirectamente y que influye en su conducta. Es decir, el clima organizacional depende principalmente de las decisiones que se tomen sobre el personal.

Al respecto, en Ecuador se conoce que los trabajadores padecen de estrés y frustración, muchas veces no son bien remunerados, el trato de los jefes no es el adecuado y no hay buena comunicación con el equipo de trabajo (Giménez, 2019). Dichas situaciones influyen negativamente en el desarrollo armónico del trabajo y amenazan la salud de los trabajadores.

Todo aquello se da a pesar de que la Constitución de la República del Ecuador en su Art. 326, numeral 5, dice: "Toda persona tendrá derecho a desarrollar sus labores en un ambiente adecuado y propicio, que garantice su salud, integridad, seguridad, higiene y bienestar" (2008). Es decir, la mayoría de las empresas ecuatorianas violan un derecho constitucional y, sobre todo, humano (ONU, 2009).

Como solución, Brancato \& Juri (2011) proponen evaluar el clima organizacional, ya que de esta manera es posible determinar las fuentes de conflicto e insatisfacción y predecir problemas críticos como: ausentismo, alta rotación, desempeño ineficiente, entre otros.

En ese sentido, se presenta el caso de una empresa atunera ecuatoriana, quien en los últimos años ha experimentado la ausencia de su personal en un $4 \%$, rotaciones de $2.5 \%$, varios inconvenientes con trabajadores y una notable reducción de la productividad.

Dadas las circunstancias, se supone que la problemática podría deberse principalmente a la ausencia de una métrica de clima laboral, puesto que esta permite conocer aspectos internos (en condiciones formales o informales) que dependen del comportamiento de los trabajadores (Machorro, Rosado, \& Romero, 2011). Es decir, que los empleadores logran conocer cómo piensan y laboran los empleados de acuerdo con el trabajo asignado (Sánchez, 2015). De esta manera es posible regular y ascender la motivación y el rendimiento profesional. Además de facilitar la construcción de una mejor estrategia organizacional para contribuir con la visión de la empresa (Segredo, 2013).

Por tanto, el presente estudio tiene como objetivo determinar las dimensiones que intervienen en el clima organizacional de la empresa atunera ecuatoriana e influyen en su productividad, mediante un cuestionario adaptado 
a la teoría de seis dimensiones del clima organizacional que propuso Weisbord (1976).

\section{MARCO TEÓRICO}

\section{Desarrollo del ser humano}

Para entender el comportamiento humano, es necesario comprender cuáles son los factores que inciden en su desarrollo y este se debe a dos fuerzas conjugadas: interna y externa. La fuerza interna proviene de una serie de etapas biológicas y código genético; mientras que la externa proviene de vivencias experimentadas en el ambiente de carácter social (Pérez \& Navarro, 2011). Estas fuerzas influyen de tal manera que los pensamientos cambian en torno a estas (Stassen, 2007).

\section{Comportamiento humano}

El comportamiento humano se encuentra determinado por un componente fisiológico que se relaciona a la parte psicológica y esta no puede separarse del entorno donde el ser humano se desenvuelve. De manera que el entorno influye directamente en el comportamiento humano (Pérez \& Vergara, 2005).

Sin embargo, no se excluye el hecho de que el comportamiento humano también dependa de capacidades, habilidades y necesidades individuales del ser humano, sumado a la realidad única que viven (Chiang, Méndez, \& Sánchez, Cómo influye la satisfacción laboral sobre el desempeño: caso empresa retail, 2010).

\section{Las relaciones humanas en el ámbito laboral}

Según Köhler y Artíles (2006), las relaciones humanas en el trabajo tienen como objetivo agrupar a colaboradores, mediante una meta y haciendo que la participación de todos sea en igualdad y considerando los intereses de todos. Es decir, se trata de una participación inclusiva.

\section{Cultura organizacional}

La cultura organizacional no se percibe $u$ observa, es algo que no se puede tocar o palpar; lo que se pude hacer es medir sus consecuencias. Esta funciona como un Iceberg: los aspectos de la parte visible son la estructura física, procesos, métodos, descripciones de puestos, etc.; mientras el lado sumergido son los aspectos no visibles y profundos que tienen un alto nivel de perceptibilidad (Chiavenato, 2011). Es decir, aun cuando la cultura no puede ser vista tangencialmente, se percibe, se vive y afecta en todos los aspectos de la organización (Duque, 2009).

Una buena cultura organizacional debe promover el desempeño laboral bajo un ambiente flexible (Madero \& Barboza, 2015), es decir, una atmosfera idónea que estimule y motive a los trabajadores (Markova, 2012). Además de implementar estrategias en cada departamento que añadan valor y éxito a la cultura (Menon, 2012).

\section{Clima organizacional}

El clima organizacional es una manifestación objetiva de la estructura de la organización que los individuos encuentran y perciben. Los aspectos objetivos del contexto son: el grado de formalización, de centralización, la tecnología, el tamaño, el lugar físico, etc.; considerando enfoques sociales, económicos y humanos (Andrade, 2015). Estas características constituyen lo que se puede llamar estructura organizacional y donde se origina del clima (Vega, Rodrigo, \& Partido, 2010).

Por otra parte, el clima laboral corresponde también a la suma de factores que componen el ambiente en el que el ser humano desarrolla su trabajo, el cual se ve influido esencialmente por la interacción humana (Gan \& Berbel, 2011). Por aquello, la interacción social debe estar por encima de los resultados al momento de plantear estrategias para alcanzar la eficiencia organizacional (Chiang, Nuñez, Martín, \& Salazar, Compromiso del Trabajador hacia su Organización y la relación con el Clima Organizacional: Un Análisis de Género y Edad, 2010).

En resumen, el clima organizacional se obtiene de la percepción del personal sobre las acciones o actuaciones de la organización, considerando que la percepción puede variar de 
individuo en individuo y que la interacción social debe primar en este clima (Alcover, Moriano, Osca, \& Topa, 2012).

\section{Importancia en el clima organizacional}

La importancia del clima organizacional radica en su capacidad de influir en el incremento de la productividad, la mitigación de ausentismos y costos, ya sean de índole interna o externa. Además de la forma en que hace que los directivos frecuentemente tengan una actitud positiva y de mejoramiento continuo, a fin de tener una organización sostenible y sustentable (Pérez \& Miranda, 2004).

A su vez, el clima recibe el impacto de los comportamientos y actitudes de los miembros de la organización, los cuales generalmente contribuyen con su propio comportamiento a que el ambiente sea agradable (Gan \& Berbel, 2011). Por el contrario, cuando existen trabajadores insatisfechos no existe una garantía de impacto positivo, sino un probable aumento de la inestabilidad laboral.

\section{Factores que afectan el clima organizacional}

Generalmente, la cantidad de horas de trabajo o descanso se consideran como indicadores del rendimiento de los trabajadores, el cual está ligado al rendimiento en productividad. Sin embargo, no son indicadores altamente fiables, puesto que son sensibles a la variación y no considera aspectos psicosociales (OIT, 2002).

Al respecto, los riesgos psicosociales laborales son situaciones que tienen una alta probabilidad de dañar de manera habitual y con gravedad la salud física, social o mental de los trabajadores (Jiménez, 2011). Por aquello, se debe priorizar la salud del personal en el ambiente laboral, mediante la asignación de horas de trabajo acorde a las leyes nacionales y de salarios justos que reflejen equidad e igualdad (Muhammad \& Wajidi, 2013).

En ese sentido, cabe enfatizar que la productividad es una medida de qué tan eficiente se utiliza trabajo o capital para producir valor económico. Por tanto, una alta productividad implica que se logra producir mucho valor económico con poco trabajo o poco capital (Galindo \& Ríos, 2015).

\section{Dimensiones del clima organizacional}

En la actualidad, se conoce de seis dimensiones desde las cuales surgen problemas relacionados al clima organizacional (Weisbord, 1976):

- Los propósitos u objetivos (conforme al tipo de negocio que se encuentren).

- Las relaciones entre las personas (el manejo de conflictos en el interior de las organizaciones).

- La estructura (la manera como se divide el trabajo en la organización).

- Los mecanismos de ayuda (tecnologías de coordinación, en términos de procesos, procedimientos, funciones, herramientas tecnológicos que ayuden el desarrollo de las actividades).

- Recompensas (el establecimiento de incentivos adecuados para el desarrollo del trabajo asignado).

- Liderazgo (en términos de cómo las personas que dirigen mantienen el equilibrio a los demás).

\section{METODOLOGÍA}

El presente estudio corresponde a un diseño cualitativo y cuantitativo, ya que se consideran opiniones de autores que estudian sobre el clima organizacional en las empresas, lo que permite dar una validez a las observaciones realizadas. Así mismo, se emplean técnicas estadísticas de procesamiento de datos con el fin de medir los resultados de las encuestas (Méndez \& Peña, 2006).

Como primer paso, se aplicó un cuestionario referente al clima organizacional, basado en la tesis doctoral propuesta por Ortega (2016). El cuestionario se ajustó al contexto de la empresa atunera en Ecuador, constando un total de 57 preguntas agrupadas en las 6 dimensiones propuestas por Wisbord (1976): 
1. Propósitos u Objetivos

2. Relaciones entre los miembros

- Comunicación

- Trato interpersonal

- Manejo de conflictos

3. La estructura y mecanismos de soporte de la Gestión

- Espacios infraestructura y lugar de trabajo

- Estructura, jerarquía y responsabilidades

4. Mecanismos de apoyo

- Tecnologías de soporte y herramientas tecnológicas

- Procesos, procedimientos y funciones

5. Recompensas

6. Liderazgo

Como opciones respuesta en cada pregunta se estipuló la escala de Likert del 1 al 5, donde:

- $1=$ Muy en desacuerdo

- 2 = Desacuerdo

- $3=$ Indiferente

- $4=$ De acuerdo

- 5 = Muy de acuerdo

La empresa cuenta con una plantilla de personal de 1674, que incluyen obreros, técnicos, administrativos y responsables de área. De dicha población se tomó una muestra considerando un 95\% de confianza, $2 \%$ de precisión, $5 \%$ de proporción y un ajuste a pérdidas del $15 \%$, lo que resultó en 422 individuos, a quienes posteriormente se les envió el cuestionario.

El cuestionario fue diseñado y difundido mediante la herramienta informática QuestionPro y correo electrónico. Cabe mencionar que este no contenía preguntas referentes a la identidad de los participantes con el fin de salvaguardarla.

Luego, se procedió a realizar un análisis de datos demográficos, así como de fiabilidad por cada una de las dimensiones que componen el clima organizacional y que fueron consideradas en el cuestionario. Para dicho análisis se utilizó el programa SPSS versión 22.

\section{RESULTADOS}

\section{Análisis demográfico}

En lo que respecta al género de los encuestados, se denota que no existe una distribución equitativa, ya que casi el $60 \%$ del personal corresponde al género masculino (ver tabla 1). En términos de equidad, lo ideal sería que exista cantidades igualitarias entre ambos géneros.

\section{TABLA 1}

\section{Género}

\begin{tabular}{lrrrr}
\hline Variables & $\begin{array}{c}\text { Fr. } \\
\text { absoluta } \\
\text { ni }\end{array}$ & $\begin{array}{c}\text { Fr. } \\
\text { relativa } \\
\text { fi }\end{array}$ & $\begin{array}{c}\text { Fr. } \\
\text { absoluta } \\
\text { acum. Ni }\end{array}$ & $\begin{array}{r}\text { Fr. } \\
\text { absoluta } \\
\text { acum. Fi }\end{array}$ \\
\hline FEMENINO & 175 & $41 \%$ & 175 & $41 \%$ \\
MASCULINO & 247 & $59 \%$ & 447 & $100 \%$ \\
\hline TOTAL & 422 & $100 \%$ & & \\
\hline
\end{tabular}

Fuente: Los autores.

En la tabla 2 se expone la edad de los encuestados por intervalos y de esta se observa que el $75 \%$ de ellos corresponde a personal joven, de manera que se puede inferir que la empresa dispone de oportunidades laborales para personas que no tienen mucha experiencia, pero poseen conocimientos frescos respecto a diversas áreas del entorno actual.

TABLA 2 Edad

\begin{tabular}{lrrrr}
\hline Variables & $\begin{array}{c}\text { Fr. } \\
\text { absoluta } \\
\text { ni }\end{array}$ & $\begin{array}{c}\text { Fr. } \\
\text { relativa } \\
\text { fi }\end{array}$ & $\begin{array}{c}\text { Fr. } \\
\text { absoluta } \\
\text { acum. Ni }\end{array}$ & $\begin{array}{c}\text { Fr. } \\
\text { absoluta } \\
\text { acum. Fi }\end{array}$ \\
\hline 18 & 7 & $2 \%$ & 7 & $2 \%$ \\
19 a 38 & 317 & $75 \%$ & 324 & $77 \%$ \\
39 a 53 & 86 & $20 \%$ & 410 & $97 \%$ \\
54 en & 12 & $3 \%$ & 422 & $100 \%$ \\
adelante & 422 & $100 \%$ & & \\
\hline TOTAL & & & & \\
\hline
\end{tabular}

Fuente: Los autores.

La hipótesis antes mencionada se confirma con los resultados obtenidos sobre el nivel académico concluido de los encuestados, los cuales se exponen en la tabla 3 . En esta se observa que $93 \%$ del personal ha concluido la primaria o la secundaria, es decir, la empresa ofrece muchas oportunidades de trabajo a bachilleres e incluso a quienes todavía no alcanzan ese nivel; siendo una medida empática e inclusiva. 
TABLA 3

Nivel académico concluido

\begin{tabular}{lrrrr}
\hline Variables & $\begin{array}{c}\text { Fr. } \\
\text { absoluta } \\
\text { ni }\end{array}$ & $\begin{array}{c}\text { Fr. } \\
\text { relativa } \\
\text { fi }\end{array}$ & $\begin{array}{c}\text { Fr. } \\
\text { absoluta } \\
\text { acum. Ni }\end{array}$ & $\begin{array}{c}\text { Fr. } \\
\text { absoluta } \\
\text { acum. Fi }\end{array}$ \\
\hline Primaria & 200 & $47 \%$ & 200 & $47 \%$ \\
$\begin{array}{l}\text { Secundaria } \\
\text { Tercer }\end{array}$ & 195 & $46 \%$ & 395 & $93 \%$ \\
nivel & 23 & $6 \%$ & 418 & $99 \%$ \\
$\begin{array}{l}\text { Cuarto } \\
\text { nivel }\end{array}$ & 4 & $1 \%$ & 422 & $100 \%$ \\
$\begin{array}{l}\text { Sin } \\
\text { estudios }\end{array}$ & 0 & $0 \%$ & 422 & $100 \%$ \\
\hline TOTAL & 422 & $100 \%$ & & \\
\hline
\end{tabular}

TABLA 4

Área de trabajo

\begin{tabular}{|c|c|c|c|c|}
\hline Grupo & Variables & Población & $\begin{array}{c}\text { Fr. } \\
\text { absoluta } \\
\text { ni }\end{array}$ & $\begin{array}{c}\text { Fr. } \\
\text { relativa } \\
\text { fi }\end{array}$ \\
\hline \multirow{11}{*}{ 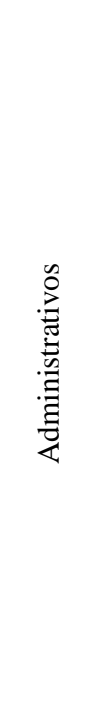 } & $\begin{array}{l}\text { Auditoría } \\
\text { Interna }\end{array}$ & 3 & 0 & $0 \%$ \\
\hline & Comercial & 38 & 2 & $0.52 \%$ \\
\hline & Compras & 10 & 0 & $0 \%$ \\
\hline & $\begin{array}{l}\text { Dispensario } \\
\text { Médico }\end{array}$ & 7 & 0 & $0 \%$ \\
\hline & Finanzas & 23 & 3 & $0.91 \%$ \\
\hline & $\begin{array}{l}\text { Gerencia de } \\
\text { Operaciones P. }\end{array}$ & 1 & 1 & $0.98 \%$ \\
\hline & $\begin{array}{l}\text { Gerencia } \\
\text { General }\end{array}$ & 10 & 3 & $1.37 \%$ \\
\hline & Legal & 1 & 0 & $0 \%$ \\
\hline & $\mathrm{O} \& \mathrm{M}$ & 3 & 0 & $0 \%$ \\
\hline & Sistemas & 14 & 2 & $0.52 \%$ \\
\hline & Talento Human & 20 & 5 & $1.30 \%$ \\
\hline \multirow{8}{*}{ 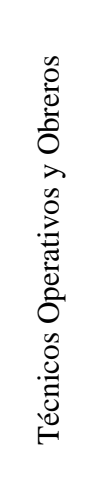 } & Bodega General & 35 & 2 & $0.52 \%$ \\
\hline & Frigorífico & 33 & 3 & $0.91 \%$ \\
\hline & $\begin{array}{l}\text { Gestión de } \\
\text { Calidad }\end{array}$ & 60 & 5 & $0.85 \%$ \\
\hline & $\begin{array}{l}\text { Logística y } \\
\text { Operaciones }\end{array}$ & 20 & 3 & $0.78 \%$ \\
\hline & Mantenimiento & 109 & 5 & $1.30 \%$ \\
\hline & Producción & 1262 & 381 & $88.61 \%$ \\
\hline & SIAM & 18 & 3 & $0.85 \%$ \\
\hline & Villas & 7 & 2 & $0.59 \%$ \\
\hline TOTAI & & 1674 & 422 & $100 \%$ \\
\hline
\end{tabular}

En referencia a lo anterior, la tabla 4 muestra el área en que mayormente la población juvenil se ve involucrada y esta es el área de producción, la cual constituye una base fundamental para la razón de ser de la empresa. Así mismo, es el área en donde se original la principal actividad económica de la entidad.

Por otro lado, los resultados obtenidos respecto al tiempo de trabajo o años de labor en la empresa permiten comprender la apertura hacia las personas jóvenes y esto podría deberse a la alta rotación, ya que el $32 \%$ dura entre 0 a 1 año en la empresa, tal como se muestra en la tabla 5:

TABLA 5

Tiempo de trabajo

\begin{tabular}{lrrrr}
\hline Variables & $\begin{array}{c}\text { Fr. } \\
\text { absoluta } \\
\text { ni }\end{array}$ & $\begin{array}{c}\text { Fr. } \\
\text { relativa } \\
\text { fi }\end{array}$ & $\begin{array}{c}\text { Fr. } \\
\text { absoluta } \\
\text { acum. Ni }\end{array}$ & $\begin{array}{c}\text { Fr. } \\
\text { absoluta } \\
\text { acum. Fi }\end{array}$ \\
\hline 0 a 1 año & 133 & $32 \%$ & 133 & $32 \%$ \\
2 a 3 años & 78 & $18 \%$ & 211 & $50 \%$ \\
4 a 8 años & 97 & $23 \%$ & 308 & $73 \%$ \\
9 años en & 114 & $27 \%$ & 422 & $100 \%$ \\
adelante & T22 & $100 \%$ & & \\
\hline TOTAL & 422 &
\end{tabular}

Fuente: Los autores.

\section{Análisis con relación al instrumento}

En la tabla 6 se muestran los resultados de las propiedades psicométricas de la escala del clima organizacional de la empresa en estudio, de la cual resultó un coeficiente de 0.950 que corresponde al Alfa de Cronbach general, considerando que la escala se muestra con un grado de fiabilidad aceptable. De esta forma se pudo establecer que los constructos de estudios se estipulan altamente confiables. 
TABLA 6

\section{Propiedades psicométricas de la escala del clima organizacional aplicada a una empresa atunera en Ecuador}

\begin{tabular}{|c|c|c|c|c|c|}
\hline $\begin{array}{c}\text { Análisis de } \\
\text { Fiabilidad por } \\
\text { Dimensiòn }\end{array}$ & KMO & $\begin{array}{c}\text { Chi-cuadrado } \\
\text { aproximado }\end{array}$ & $\mathrm{gl}$ & $\begin{array}{c}\text { Alfa de } \\
\text { Cronba } \\
\text { ch }\end{array}$ & $\begin{array}{c}\mathrm{N}^{\mathrm{o}} \text {. de } \\
\text { elementos }\end{array}$ \\
\hline $\begin{array}{l}\text { Propósitos y } \\
\text { objetivos }\end{array}$ & 0.789 & 595.461 & 28 & 0.744 & 8 \\
\hline $\begin{array}{l}\text { Relaciones } \\
\text { entre los } \\
\text { miembros }\end{array}$ & 0.905 & 1832.516 & 91 & 0.852 & 14 \\
\hline La estructura & & & & & \\
\hline $\begin{array}{l}\text { y } \\
\text { mecanismos } \\
\text { de soporte de } \\
\text { la gestión }\end{array}$ & 0.869 & 1013.067 & 36 & 0.843 & 9 \\
\hline $\begin{array}{l}\text { Mecanismos } \\
\text { de apoyo }\end{array}$ & 0.889 & 1394.081 & 55 & 0.850 & 11 \\
\hline Recompensas & 0.814 & 549.834 & 15 & 0.770 & 6 \\
\hline Liderazgo & 0.852 & 791.715 & 36 & 0.791 & 9 \\
\hline \multicolumn{4}{|c|}{ ESCALA TOTAL } & 0.950 & 57 \\
\hline
\end{tabular}

Fuente: Los autores.

Por otro lado, considerando que la encuesta contiene seis dimensiones y que la segunda y cuarta dimensión contienen subdimensiones; en la tabla 7 se detalla las subdivisiones y sus ítems:

\section{TABLA 7}

\section{Distribución de dimensiones según ítems}

\begin{tabular}{|c|c|c|}
\hline$\#$ & Ítem & Dimensiones \\
\hline 8 & CLOB & Propósitos u Objetivos \\
\hline 5 & CREC & Relaciones entre los miembros - Comunicación \\
\hline 5 & CRET & $\begin{array}{l}\text { Relaciones entre los miembros - Trato } \\
\text { interpersonal }\end{array}$ \\
\hline 4 & CREM & $\begin{array}{l}\text { Relaciones entre los miembros - Manejo de } \\
\text { conflictos }\end{array}$ \\
\hline 4 & CESES & $\begin{array}{l}\text { La estructura y mecanismos de soporte de la } \\
\text { gestión - Espacios, infraestructura y lugar de } \\
\text { trabajo }\end{array}$ \\
\hline 5 & CESTR & $\begin{array}{l}\text { La estructura y mecanismos de soporte de la } \\
\text { gestión - Estructura, jerárquica y } \\
\text { responsabilidades }\end{array}$ \\
\hline 6 & CMAT & $\begin{array}{l}\text { Mecanismos de apoyo - Tecnologías de } \\
\text { soporte y herramientas tecnológicas }\end{array}$ \\
\hline 5 & CMAP & $\begin{array}{l}\text { Mecanismos de apoyo - Procesos, } \\
\text { procedimientos y funciones }\end{array}$ \\
\hline 6 & CRCM & Recompensas \\
\hline
\end{tabular}
Fuente: Los autores.

Se emplearon los ítems a fin de sintetizar y mejorar la visualización de las tablas de resultados. Es así como, conociendo el significado de que cada ítem, se puede comprender con facilidad la tabla 8 (ver apéndice), la cual muestra los estadísticos descriptivos del clima organizacional.

De dicha tabla se denota que existen tres dimensiones que presentan notables inconformidades por parte del personal, estas refieren a la estructura de liderazgo, objetivos y recompensas que posee la organización, con un $41 \%, 45 \%$ y $52 \%$, respectivamente.

Específicamente, el personal se mostró inconforme con la forma en que se lleva a cabo el trabajo en equipo y la cantidad de recursos que le disponen para laborar efectivamente. Así mismo, percibe de manera negativa el cumplimiento de objetivos en relación con la misión y el ajuste presupuestario respecto a recursos. Por último, siente que los salarios y beneficios adicionales no se ajustan a sus necesidades.

Sin embargo, también hay dimensiones que satisfacen las expectativas o necesidades del personal, representando más del $60 \%$ de conformidad y estas son:

- Relaciones entre los miembros: comunicación con $73 \%$, trato interpersonal con $78 \%$ y manejo de conflictos con $85 \%$.

- Estructura y Mecanismos de Soporte de la Gestión: espacios infraestructura y lugar de trabajo con $81 \%$ y, estructura, jerarquía y procedimientos con $72 \%$.

- Mecanismos de Apoyo: tecnologías de soporte y herramientas tecnológicas con $68 \% \mathrm{y}$, procesos, procedimientos $\mathrm{y}$ funciones con $64 \%$.

Respecto a las relaciones entre los miembros, el personal mostró mayor conformidad con la eficiencia de la comunicación por parte de los jefes inmediatos, así como de la ayuda que recibe de estos en sus actividades. Pero su conformidad fue aún más notable sobre la forma en que se resuelven los conflictos, es decir, a través del diálogo.

En cuanto a la estructura y mecanismos de soporte de la gestión, el personal respondió positivamente sobre las instalaciones donde 
labora, así también sobre la estructura organizacional.

Por último, respecto a los mecanismos de apoyo, se denotó mayor conformidad sobre las capacitaciones que los trabajadores reciben y la claridad de las funciones y procedimientos.

\section{CONCLUSIONES}

De los resultados se concluye que es una medida positiva que la empresa de apertura laboral a los jóvenes bachilleres, sin embargo, sería mucho más beneficioso que les brinden estabilidad laboral, es decir, más de un año de labor. Así mismo, se debería incrementar la participación de la mujer en el área de producción. En la medida en que esto se cumpla, la empresa podrá mejorar su clima e imagen, volviéndola más atractiva tanto para accionistas como para consumidores. Pero podrá mejorar aún más sus niveles de producción, ya que el comportamiento del empleado es recíproco al comportamiento de la organización con este (Pons \& Ramos, 2012).

Por otro lado, los resultados también permiten considerar como puntos de mejora todos los aspectos relacionados al liderazgo, objetivos y sistema de recompensas. Al ser estas dimensiones las menos acordes a la satisfacción del personal, se denota que la empresa no trabaja alineada a sus objetivos, sino que prioriza generar capital suficiente para cubrir sus gastos. Con la implementación de un buen liderazgo corporativo es posible redirigir el trabajo al cumplimiento de objetivos, sin dejar de considerar el pago justo al personal. Por el contrario, dentro de los objetivos se debería considerar un sistema de recompensas justo que eleve la motivación y compromiso de los empleados (Chiang, Nuñez, Martín, \& Salazar, 2010).

Dada la fiabilidad del estudio y los beneficios que se puede obtener de este, se recomienda la aplicación de este de forma periódica, ya que de esta manera sería posible realizar comparaciones y evaluar las fortalezas y debilidades de la empresa a través del tiempo. Incluso anticiparse a problemas y fortalecer las medidas de control de operaciones (Rodríguez, 2015).
Finalmente, cabe mencionar que el presente estudio corresponde al primero aplicado a una empresa atunera ecuatoriana, resultando con un $95 \%$ de fiabilidad. De manera que es posible que este se replique en otras empresas industriales y que, a futuro, el clima organizacional también sea considerado como una métrica de competitividad en el mercado.

\section{REFERENCIAS}

Alcover, C., Moriano, J., Osca, A., \& Topa, G. (2012). Psicología del trabajo. Madrid: UNED.

Andrade, M. (2015). El Clima Organizacional Y Su Incidencia En El Desempeño Laboral Del Personal Docente De La Unidad Educativa "José María Velasco Ibarra" De La Ciudad De Milagro. Guayaquil: Universidad Laica Vicente Rocafuerte de Guayaquil.

Brancato, B., \& Juri, F. (2011). ¿PUEDE INFLUIR EL CLIMA LABORAL EN LA PRODUCTIVIDAD? UNCUYO.

Chiang, M., Méndez, G., \& Sánchez, G. (2010). Cómo influye la satisfacción laboral sobre el desempeño: caso empresa retail. Theoría, 19(2), 21-36.

Chiang, M., Nuñez, A., Martín, M., \& Salazar, M. (2010). Compromiso del Trabajador hacia su Organización y la relación con el Clima Organizacional: Un Análisis de Género y Edad. Panorama Socieconómico, 90-100.

Chiang, M., Nuñez, A., Martín, M., \& Salazar, M. (2010). Compromiso del Trabajador hacia su Organización y la relación con el Clima Organizacional: Un Análisis de Género y Edad. Panorama Socieconómico, 90-100.

Chiavenato, I. (2011). Administración de Recursos Humanos El capital humano de las organizaciones. Mc Graw Hill.

Constituyente, A. (2008). Constitución de la República del Ecuador. Quito, Pichincha, Ecuador.

Corredor, A. (2016). PYMES: GESTIÓN Y CLIMA ORGANIZACIONAL. Barranquilla: Coruniamericana. Obtenido de https://books.google.com.ec/books?id=SWB 
gDwAAQBAJ\&printsec $=$ copyright $\# \mathrm{v}=$ onepa ge $\& q \& f=$ false

Duque, M. (2009). Desarrollo del modelo del clima organizacional MAQUIAVÍCOLA LTDA. Pontificia Universidad Javeriana, Bogotá.

Galindo, M., \& Ríos, V. (2015). Productividad. México ¿Cómo vamos? Obtenido de https://scholar.harvard.edu/files/vrios/files/20 1508_mexicoproductivity.pdf

Gan, F., \& Berbel, G. (2011). Manual de Recursos Humanos. Barcelona: UOC.

Giménez, J. (2019). Trabajadores ecuatorianos explotados como hace 200 años. El País. Obtenido de https://elpais.com/elpais/2019/02/14/planeta_ futuro/1550142855_619700.html

Hall, H. (2009). Cultura Corporativa y Productividad Organizacional. Prentice-Hall Hispanoamérica.

Jiménez, B. (2011). Factores y riesgos laborales psicosociales: conceptualización, historia y cambias actuales. Med Segur Trab, 57(1), 119.

Köhler, H., \& Artíles, A. (2006). Manual de la socialogia del trabajo y de las relaciones laborales. Madrid: Delta Publicaciones.

Machorro, F., Rosado, M., \& Romero, M. (2011). Diseño de un instrumento para evaluar el clima organizacional en un complejo petroquímico del Estado de Veracruz. Ciencias Administrativas, 30-35.

Madero, S., \& Barboza, G. (2015). Interrelación de la cultura, flexibilidad laboral, alineación estratégica, innovación y rendimiento empresarial. México: Universidad Autónoma de México.

Markova, G. (2012). Building dynamic capabilities: The case of HRIS. Management Research, 81-98.

Méndez, L., \& Peña, J. (2006). Manual práctico para el diseño de la Escala Likert. México: Trillas.

Menon, S. (2012). Human resource practices, supply chain performance, and wellbeing.
International Journal of Manpower, 769785 .

Muhammad, R., \& Wajidi, F. (2013). Factors influencing job satisfaction in public health sector. Global Journal of Management and Business Research, 61-65.

OIT, O. I. (2002). La evolución del empleo, el tiempo de trabajo y la formación en la industria minera. Oficina Internacional de Trabajo, Ginebra. Obtenido de https://www.ilo.org/public/libdoc/ilo/2002/10 2B09_226_span.pdf

ONU, N. U. (2009). Salud y vida en el trabajo: Un derecho humano fundamental. Oficina Internacional del Trabajo, Ginebra. Obtenido de

https://www.ilo.org/legacy/english/protection /safework/worldday/products09/booklet_09es.pdf

Ortega, J. (2016). Clima organizacional: origen evolución y medición. Universidad del Rosario, Bogotá. Obtenido de https://pure.urosario.edu.co/es/publications/cl ima-organizacional-origenevoluci\%C3\%B3n-y-medici\%C3\%B3n

Pérez, A., \& Miranda, D. (2004). Clima organizacional en salud pública. Consideraciones generales. Obtenido de http://www.cocmed.sld.cu/no83/n83rev4.htm

Pérez, M., \& Vergara, M. (2005). Ciencias psicosociales aplicadas. Barcelona: Universitat de Barcelona.

Pérez, N., \& Navarro, I. (2011). Psicología del desarrollo humano: del nacimiento a la vejez. Club Universitario.

Pons, F., \& Ramos, J. (2012). Influencia de los Estilos de Liderazgo y las prácticas de Gestión de RRHH sobre el Clima Organizacional de Innovación. Revista de Psicología del Trabajo y de las Organizaciones, 81-98.

Rodríguez, A. (marzo de 2015). Escuela de Organización Industrial. Obtenido de https://www.eoi.es/blogs/mintecon/2015/03/2 6/clima-organizacional/ 
Sánchez, J. (2015). Diagnóstico del Clima Organizacional en la Dirección Provincial del Banco de Crédito y Comercio de Holguin. México: Grin Verlag.

Segredo, A. (2013). Clima organizacional en la gestión del cambio para el desarrollo de la organización. Revista Cubana de Salud Pública, 385-393.

Stassen, K. (2007). Psicología del Desarrollo. Infancia y adolescencia. Madrid: Médica Panamericana.

Vega, M., Rodrigo, M., \& Partido, A. (2010). Relaciones entre el clima organizacional y la satisfacción laboral. Madrid: Universidad de Comillas.

Weisbord, M. (1 de diciembre de 1976).

Organizational Diagnosis: Six Places To Look for Trouble with or Without a Theory. Group \& Organization Studies, 1(4), 430447. Obtenido de https://doi.org/10.1177/10596011760010040 5 


\section{APÉNDICES}

TABLA 8

Estadísticos descriptivos de clima organizacional

\begin{tabular}{|c|c|c|c|}
\hline Ítems & Media & Desviación estándar & $\begin{array}{c}\text { Promedio } \\
\text { dimensional }\end{array}$ \\
\hline CLOB 1 & 3.64 & 1.069 & \multirow{7}{*}{$45 \%$} \\
\hline CLOB 2 & 3.01 & 1.434 & \\
\hline CLOB 3 & 3.30 & 1.197 & \\
\hline CLOB 4 & 3.34 & 1.227 & \\
\hline CLOB 5 & 2.36 & 1.438 & \\
\hline CLOB 6 & 3.82 & 1.102 & \\
\hline CLOB 7 & 2.92 & 1.471 & \\
\hline CLOB 8 & 3.61 & 1.218 & \multirow{7}{*}{$73 \%$} \\
\hline CREC 1 & 3.67 & 1.167 & \\
\hline CREC 2 & 3.68 & 1.178 & \\
\hline CREC 3 & 3.32 & 1.131 & \\
\hline CREC 4 & 3.64 & 1.199 & \\
\hline CREC 5 & 3.30 & 1.328 & \\
\hline CRET 1 & 3.88 & 1.070 & \\
\hline CRET 2 & 3.62 & 1.146 & \multirow{3}{*}{$78 \%$} \\
\hline CRET 3 & 3.17 & 1.271 & \\
\hline CRET 4 & 3.52 & 1.236 & \\
\hline CRET 5 & 3.43 & 1.209 & \multirow{6}{*}{$85 \%$} \\
\hline CREM 1 & 3.38 & 1.197 & \\
\hline CREM 2 & 3.41 & 1.170 & \\
\hline CREM 3 & 3.33 & 1.268 & \\
\hline CREM 4 & 2.82 & 1.304 & \\
\hline CESES 1 & 3.24 & 1.234 & \\
\hline CESES 2 & 3.91 & 1.138 & \multirow{2}{*}{$81 \%$} \\
\hline CESES 3 & 3.31 & 1.233 & \\
\hline CESES 4 & 3.46 & 1.148 & \multirow{7}{*}{$72 \%$} \\
\hline CESTR 1 & 3.62 & 1.063 & \\
\hline CESTR 2 & 3.77 & 1.078 & \\
\hline CESTR 3 & 3.75 & 1.121 & \\
\hline CESTR 4 & 3.63 & 1.039 & \\
\hline CESTR 5 & 3.69 & 1.128 & \\
\hline CMAT 1 & 4.08 & 1.133 & \\
\hline CMAT 2 & 4.05 & 1.094 & \multirow{5}{*}{$68 \%$} \\
\hline CMAT 3 & 3.63 & 1.122 & \\
\hline CMAT 4 & 3.69 & 1.008 & \\
\hline CMAT 5 & 3.71 & 1.009 & \\
\hline CMAT 6 & 3.64 & 1.060 & \\
\hline
\end{tabular}




\begin{tabular}{|c|c|c|c|}
\hline Ítems & Media & Desviación estándar & $\begin{array}{c}\text { Promedio } \\
\text { dimensional }\end{array}$ \\
\hline CMAP 1 & 3.68 & 1.078 & \multirow{6}{*}{$74 \%$} \\
\hline CMAP 2 & 3.53 & 1.053 & \\
\hline CMAP 3 & 3.54 & 1.030 & \\
\hline CMAP 4 & 3.44 & 1.043 & \\
\hline CMAP 5 & 2.97 & 1.110 & \\
\hline CRCM 1 & 3.12 & 1.267 & \\
\hline CRCM 2 & 3.12 & 1.142 & \multirow{5}{*}{$52 \%$} \\
\hline CRCM 3 & 2.67 & 1.280 & \\
\hline CRCM 4 & 3.41 & 1.088 & \\
\hline CRCM 5 & 3.14 & 1.260 & \\
\hline CRCM 6 & 3.05 & 1.265 & \\
\hline CLID 1 & 3.66 & 1.118 & \multirow{9}{*}{$41 \%$} \\
\hline CLID 2 & 3.19 & 1.126 & \\
\hline CLID 3 & 3.19 & 1.304 & \\
\hline CLID 4 & 2.81 & 1.319 & \\
\hline CLID 5 & 3.86 & 1.157 & \\
\hline CLID 6 & 3.30 & 1.215 & \\
\hline CLID 7 & 3.39 & 1.143 & \\
\hline CLID 8 & 3.65 & 1.068 & \\
\hline CLID 9 & 3.63 & 1.158 & \\
\hline
\end{tabular}

